\title{
Detection of Steganography Using Convolution Neural Network Machine Learning Method Over Wireless Network
}

\author{
Devishree Naidu ${ }^{* *}$, and Shubhangi Tirpude ${ }^{2}$ \\ ${ }^{1,2}$ Shri Ramdeobaba College of Engineering and Management, India
}

\section{ABSTRACT}

Steganography is the science of storing information secretly by embedding it in media (e.g. Images). Steganalysis can apparently be described as the opposite of Steganography. The strength of steganography algorithms is a trade of how well steganalysis of the encrypted file is done. Steganalysis algorithms helps in improving the image robustness to resist detection of steganography. Traditional approaches of detection are mostly human based. There exists several steganalysis consisting of image examinations or humanoid graphic that includes inspection for detecting whether a file contains hidden message using steganography algorithm. These approaches are very delicate, a miniscule change in algorithm can render a steganalysis approach useless. An approach of steganalysis using machine learning algorithms can foresee the capabilities of human observation skills to be a harbinger for steganalysis. launching dictionary attacks for such framework is quite communal this includes knowledge of distributed computing. In Machine Learning approach, canvas formally used as representation form of any media file wherein available spaces can be used to hide informative messages. The distinguishable features get improved from clean to stego-bearing files which then goes under processing. The proposed method seamlessly explores the use of machine learning CNN scheme that are used to distinguish clean and stego-bearing files.

\section{KEY WORDS: STEGANALYSIS, STEGANOGRAPHY, DISCRETE WAVELET TRANSFORMATION, MARKOV CHAINS, CNN.}

\section{INTRODUCTION}

Many investigations are closely deliberately conveying that terrorists are using steganography to hide their communication from law enforcement. There isn't a way to verify these claims as per technical information is concerned. However, number of new sources intellect about hiding of messages inside images that are posted on internet public sales sites like Amazon, FlipKart, or eBay. Detecting and preventing data leakage is one of the most important things. Especially, in terms of corporate

\section{ARTICLE INFORMATION}

Received 14th Oct 2020 Accepted after revision 28th Dec 2020 Print ISSN: 0974-6455 Online ISSN: 2321-4007 CODEN: BBRCBA

Thomson Reuters ISI Web of Science Clarivate Analytics USA and Crossref Indexed Journal

\section{Clarivate
Analytics}

NAAS Journal Score 2020 (4.31) SJIF: 2020 (7.728)

A Society of Science and Nature Publication,

Bhopal India 2020. All rights reserved.

Online Contents Available at: http//www.bbrc.in/

Doi: http://dx.doi.org/10.21786/bbrc/13.14/61 networks, it is observed that the significance of this issue increased lately. Many open-source Data Prevention solutions are used to prevent data from thieves.

Steganography is the way to hide the secret messages. Messages can be hidden in images or any other digital objects. To any unpremeditated spectator inspecting these steganographic images, the messages are invisible. It seems to be similar to cryptography but they are different. The most important difference is that; in cryptography, while data is being transferred, data encryption is seen by someone, but in steganography, the transferred secret data cannot be directly recognized by someone. We focus not only on hiding messages but also the hidden messages sent should go undetected. Steganography supports hiding messages amongst the huge volume of Internet traffic. The process of detecting embedded messages stored in any digital media is known as steganalysis, as identified by steganographic methods, and performing steganalysis technique this is referred as attack. It focuses on breaking 
the security of steganography. The original content of file identification and creating statistical experimental setup for signature detection evolves current state of art. The messages which are hidden are characteristically placed in images or digital-media files.

Machine Learning and Data Mining application are difficult due to its variation in size of files and its varying formats. Typically, JPEG/MPEG results into lossy compression whereas GIF uses lossless representation for efficient indexing. This makes a difference for its difficult representation. Our approach is quite appropriate to many media and makes the images or media to be used as canvas i.e. hidden messages can be made easily available to be written in the files. We can then apply Standard machine algorithms to detect steganography in both the formats as JPEG and GIF. Applying Machine Learning algorithms our experimental shows that This particular approach supports capability in detecting hidden messages in all types of file formats. It is a very difficult task to design such tools to automate pattern recognition that uses complex domain analysis. It is with various examination and several experimental observations, we analyzed that how concealed image can be detected using steganography algorithm.

Literature Review: Fast Fourier transformation forms the basis understanding of DCT method, and is known for best standard for JPEG due to its significant reasoning. One important reason is, it has capability to pack entire energy in its low frequency, image data and second one is reduced blocking effect which enables sub-image boundaries to get visible. Block pixel calculation is carried out by converting the raster data into $8 \times 8$ blocks of pixels extraction for JPEG. Each block further gets compressed by Huffman encoding, in most cases process results into Lossy Compression and it representation may allow in varying degree. It means that we cannot recreate the exact original image, typically its due to loss of information while applying compression algorithm. DCT Transformation can lead to different levels of compression owing to its varying severity. In comparison we follow another technique as optimized Discrete wavelet Transform where decomposed image undergoes with manipulating the transform coefficient to hide the secret information that are also subjected to various types of attack related to image processing.

A. Technique for Image Embedding: All types of traditional image steganography methods are mostly based on embedding technique. We can embed the secret information using particular image carrier by applying some modification, the main focus of our research is to keep embedding distortion to be at minimum level and improving its capacity by means of technique such as least significant matching (LSB) as proposed. Many techniques are into practice, they include highly undetectable stego (HUSG0) and subtractive pixel adjacent Matrix (SPAM) representation. These methods have been designed principally based on minimum distortion function. It is usually according to the weighted total summation of vector feature image, extracted from covered image and its modified one. A scheme called as wavelet obtained weight (WOW) that involve maximum gain of covered images including rule-based texture modification with more pixel value. A source-based technique named as S-UNIWARD also works in a similar manner with noisy based multifaceted surface section. In the existing internet communication, much of confidential information needs rigorous protection and with the advent of larger threats that are concerned with origin content under embedding technique, the steganalysis capability have been improved a lot by using Machine learning approaches and the way statistical analysis gets performed.

B. Expansion of Steganalysis: An extension of the spatial rich model for the steganalysis of color images a novel approach of SWE based on deep convolution adversarial network has been introduced by mapping secret into noise vector and thereby giving training to network model for generating carrier image based on noise vector using extractor.. As a security concern sometimes encrypted images hidden message on the cloud can undergo steganalysis, in such cases authentication dependencies for retrieval of information may change the entire embedding that creates a noise and are difficult to detect. Stego signal are very much similar to noise like pattern which can be captured from learned illustration for feature visualization in such cases deep models are preferrable to be more focused onto steganalysis automation. A hybrid deep-learning framework is proposed for JPEG image steganalysis that makes use of three phases as Convolution, Quantization, and Truncation for training model parameters can be applied to confidential message for authentication as an extension.

Figure 3: Flow Control of proposed methodology

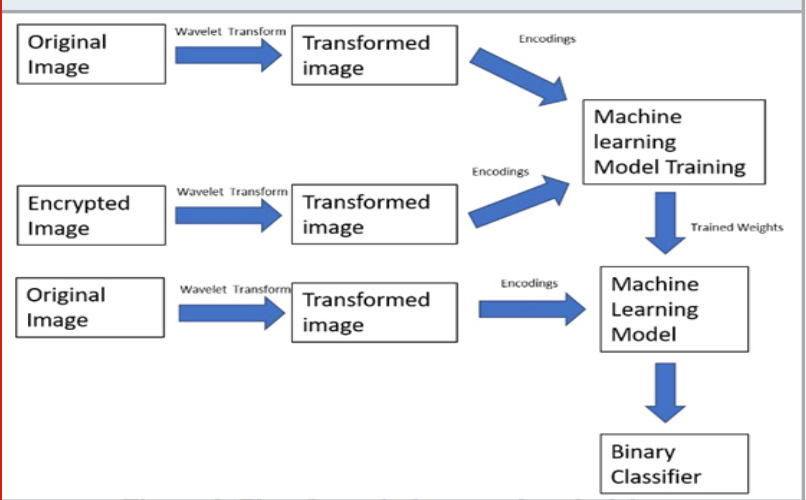

Proposed Approach: The first and important part of our approach contains application of wavelet transformation on the provided image and its non-processed image. From this we can obtain an assumption of the deviation occurring in the steganography. The wavelet transform $(W T)$ uses a type of mapping from L2(R) $\rightarrow$ L2(R2), if we compare it with spaced time-frequency localization, wavelet transform, the time frequency is most superior where transform invertibility is more focused. Normally it follows same algorithm used for 1D as 2-D wavelet for decomposition purpose. The way scaling function and 
vector wavelet works here it makes a difference. Moreover two-dimensional DWT leads to a decomposition of guesstimate coefficients at level $\mathrm{j}$ in four constituents: the approximation is at level $\mathrm{j}+1$, and the details in three orientations (horizontal, vertical, and diagonal).

The reconstruction of a signal from its transform values are dependent on unevenness grid samples but on a similar line oversampling may result into information loss for which framing concept is very much appreciated for addressing the issues. The image below in the Figure 1. portrays the basic breakdown steps for images. The design of DWT requires that scaling vector to be satisfied for the constraint of invertibility and the system applies these vectors as discrete convolution kernel for its implementation.

Figure 2: Single-level discrete 2-D wavelet transform
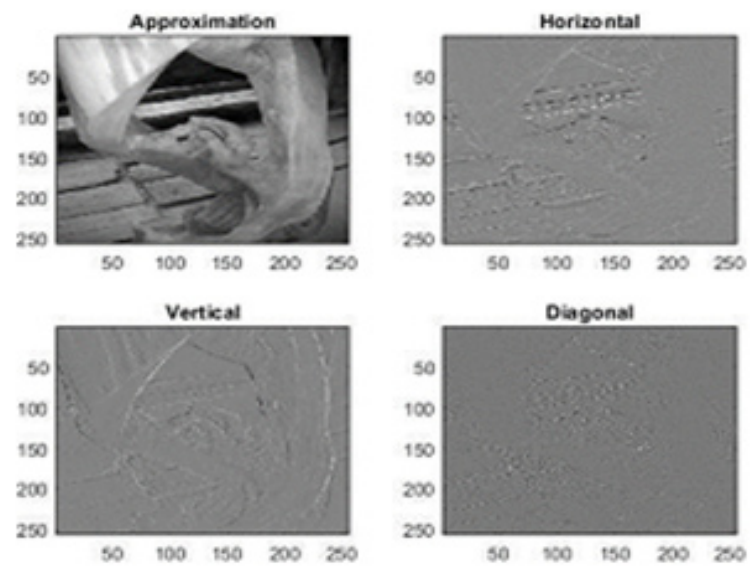

The training data would be the deviations being observed in the steganalysis. On feeding the machine learning algorithm with these data we can generate a model which can create rules to detect steganography in the given images. The model will be further annotated with a binary classifier which will confirm steganographically processed image.

Experimental Results and Analysis: The main goal of this experiment is to determine the fact that Machine learning Techniques and mining process can it be combinedly identifies whether messages are hidden using a specific steganography technique. As in Figure 3 transformation performance includes the outcome of transformation coefficient to hide the secret which involves the analysis needs to be drawn from the hard and soft threshold to get the original coefficient. Techniques required for statistical major attacks against steganography needs to be updated frequently. We can create good learning models for existing steganography by making use of datamining methods and deep learning methods. They can be trained to successfully attack on variety of steganography which includes formerly invisible disparities of existing techniques. In order to prove that steganalysis algorithm used for forensic investigation offered by CNN can also resist detection from the created stego images by the prescribe method.

Figure 5: Transformation Steganalysis Performance

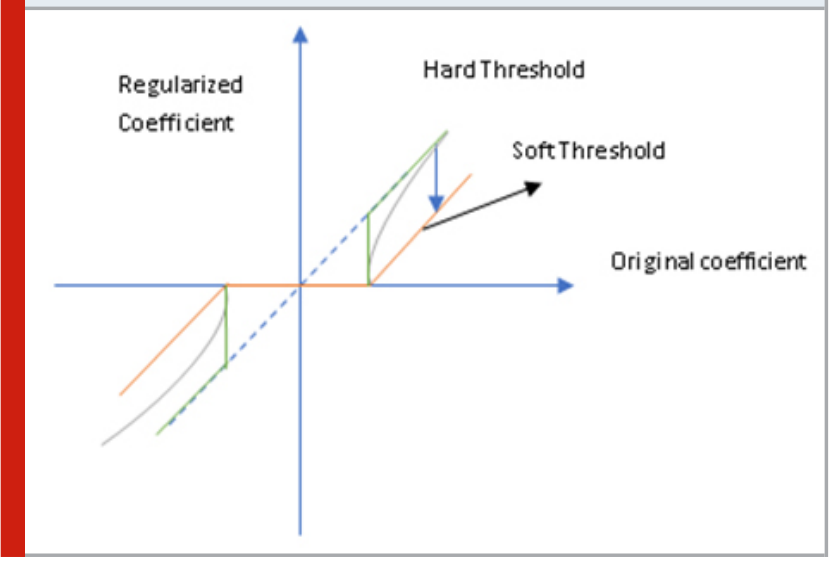

We experimented on 2D wavelet transformation images and applied machine learning based method for further binary classifier processed image and realized a unique way to detect the rules for steganalysis for better result. The method also allows to check that clean and stego bearing files are distinguishable. The detection accuracy is turned out to be 0.98 which is not low it means this approach shows good detection capability that is desired for forensic analysis.

\section{CONCLUSION}

Our approach provides the solution that mainly focuses into stego analysis related to image steganography to detect clear media files for canvas features. We consider wavelet transformation as an application. As the internet services are being available easily at cheap costs to the masses, it is becoming easier to hide information for unethical activities. On similar platform we can hide the information over videos. An extension from images to videos can be thought for further consideration. Also, the scalability and optimization of the method needs to be worked on for large scale to be used over millions of search data at a time to cover at all the ground. use unsupervised anomaly detection approaches to steganalysis. On improvisation of the model and acquisition of computing resources we can also look forward to use steganographic approaches for video steganography detection. This trivial contribution towards data breaching and data security can be materialized by acquiring more training data, with this we forestall being able to extend this work to these approaches should be able to identify and differentiate between stego bearing files and clean media files.

All this is performed without having a knowledge of steganography technique that was used and hence should also able to detect steganography hidden messages using more advanced algorithms. This conveys strongly exciting potential to surpass the current fragile nature of modern steganalysis - it may be possible to identify 
that a file has a hidden message, that is unidentifiable due to formerly unobserved steganography algorithm. Nevertheless, steganalysis and forensics methods can be used in real-world applications, by keeping our method secret for the required training set. During pandemic situation lot of medical image information that are confidential to be treated and in case there is a cyberattack then this kind of analysis is certainly be useful. In future it can be implemented with more improved learning.

\section{REFERENCES}

Devishree et al, (2020) Novel Idea of Unique Key Generation and Distribution Using Threshold Science to Enhance Cloud Security. In Smart Trends in Computing and Communications., vol 165.

D Naidu. et al. Data Hiding using Meaningful Encryption Algorithm to Enhance Data Security. http://www.warse. org/IJATCSE/static/pdf/file/ijatcse226922020.pdf.doi. org/10.30534/ijatcse/2020/226922020, Volume 9 No.2,
March -April 2020.

D. Hu, L. Wang, W. Jiang, S. Zheng and B. Li, "A Novel Image Steganography Method via Deep Convolutional Generative Adversarial Networks," in IEEE Access, vol. 6, pp. 38303-38314, 2018, doi:10.1109/ ACCESS.2018.2852771.

J. Zeng, S. Tan, B. Li and J. Huang, "Large-Scale JPEG Image Steganalysis Using Hybrid Deep-Learning Framework," in IEEE Transactions on Information Forensics and Security, vol. 13, no. 5, pp. 1200-1214, May 2018, doi: 10.1109/TIFS.2017.2779446.

L. Rathika, B. Loganathan, Approaches and Methods for Steganalysis - A Survey IJARCCE ISSN (Online) 22781021 ISSN (Print) 23195940 International Journal of Advanced Research in Computer and Communication Engineering ISO 3297:2007 Certified Vol. 6, Issue 6, June 2017, DOI 10.17148/IJARCCE.2017.6678 433 Ling Gan and Ning Sun 2019 IOP Conf. Ser.: Mater. Sci. Eng. 677022097. 